\title{
Conformación, fortalezas y debilidades de cuerpos académicos en una universidad pública del sureste de México
}

\section{Configuration, Strengths, and Weaknesses of Academic Bodies in a State University in Southeastern Mexico}

\author{
ISSN 1510-2432 - ISSN 1688-9304 (en línea) - DOI: 10.18861/cied.2017.8.2.2685
}

\begin{abstract}
Alva del Rocío Pulido Téllez
Maestría en Sistemas de Información Fundación Arturo Rosenblueth, México. Licenciada en Informática, Instituto Tecnológico de Villahermosa México. Profesora Investigadora, Universidad Juárez Autónoma de Tabasco, México. Miembro, Sistema Estatal de Investigadores de Tabasco. Reconocimiento al Mérito Académico 2006, Universidad Juárez Autónoma de Tabasco.
\end{abstract}

Débora Domínguez Pérez

Doctora en Educación, Centro Internacional de Postgrado, México. Maestra en Ciencias de la Educación, Universidad del Valle de México. Licenciada en Idiomas, Universidad Juárez Autónoma de Tabasco, México. Profesora Investigadora de Ética, Metodología de la Investigación y Lengua Extranjera, Universidad Juárez Autónoma de Tabasco. Premio al Mérito Académico 2007, División Académica de Ingeniería y Arquitectura. Reconocimiento al Perfil Deseable del Programa de Desarrollo Profesional Docente (PRODEP) 2016-2019, Secretaría de Educación Pública. México.

María del Carmen Sandoval Caraveo

Doctora en Gestión Estratégica y Políticas de Desarrollo, Universidad del Mayab, México. Maestría en Administración con especialidad en Dirección del Factor Humano, Universidad del Valle de México. Licenciada en Administración, Universidad Juárez Autónoma de Tabasco, México. Profesora Investigadora, Universidad Juárez Autónoma de Tabasco. Reconocimiento al Mérito Académico 2005 y 2015 y al Mérito Científico 2016,Universidad Juárez Autónoma de Tabasco. Miembro del Sistema Nacional de Investigadores Nivel I, Consejo Nacional de Ciencia y Tecnología México.

Fecha de recibido: 06/10/2016

Fecha de aceptado: 29/06/2017

\section{Resumen}

En los últimos años la educación superior en México ha intensificado sus esfuerzos por elevar la calidad de los procesos educativos a través del fomento de la investigación científica, la habilitación de la planta académica y el fortalecimiento de los cuerpos académicos así como de las respectivas líneas de generación y aplicación del conocimiento. La finalidad de este estudio fue describir los procesos de conformación y evolución así como las fortalezas y debilidades de los cuerpos de una facultad de ingeniería. El estudio fue descriptivo transversal con enfoque mixto. Por una parte se analizaron datos estadísticos relevantes proporcionados por la institución y se empleó la entrevista estructurada dirigida a líderes y ex líderes de dicho centro con la finalidad de recolectar sus puntos de vista. Los principales resultados indicaron que dichos cuerpos académicos presentaron limitaciones respecto a su conformación debido a que los profesores no cumplían con los requisitos para el grado de doctor ni había productos académicos desarrollados entre 
sus integrantes. Por otra parte, se hallaron avances en el número de cuerpos académicos en consolidación. La principal fortaleza identificada fue que el total de los integrantes de cada cuerpo académico cuenta con el reconocimiento del perfil deseable otorgado por el Programa para el Desarrollo Profesional Docente, al tiempo que la mayor debilidad se manifiesta en el escaso número de miembros pertenecientes al Sistema Nacional de Investigadores.

Palabras clave: profesor investigador, investigación, ingeniería, docencia, educación superior, conocimiento.

\begin{abstract}
In recent years, higher education in Mexico has intensified efforts to increase the quality of educational processes through the promotion of scientific research, the qualification of academic staffs and the strengthening of academic bodies, as well as of lines of generation and application of knowledge. The purpose of the present study is to describe the processes of configuration, and evolution as well as the strengths and weaknesses of the academic bodies of an Engineering school. It is a descriptive, cross-section study with a mixed approach. On one hand, relevant statistic data provided by the educational center were analyzed, and structured interviews were carried out with leaders and former leaders of the academic bodies in order to gather their points of view. Main findings indicated that said academic groups did not fulfill the requirements as regards the number of members with PhD degrees, nor had their members developed academic products. On the other hand, important progresses were found in the number of academic bodies that achieved On Consolidation status. The main strength identified was that the whole number of members has obtained the Recognition of Desirable Profile awarded by the Professional Teaching Development Program (PRODEP), while the main weakness lies in the low number of members admitted in the National Researchers System.
\end{abstract}

Key words: research professor, research, engineering, teaching, higher education, knowledge.

\title{
Introducción
}

Ante la necesidad de profesionalizar a los académicos de las Instituciones de Educación Superior (IES) de nuestro país, a mediados de la década de los setenta del siglo XX comenzó a difundirse una serie de programas cuyo fin era profesionalizar a los académicos que laboraban en dichas instituciones. Así fue como las políticas nacionales e institucionales fueron encaminadas a diseñar actividades que permitieran promover el diseño y operación de programas orientados a la formación de profesores para que no solo se dedicaran a su actividad básica, la docencia, sino también a actividades complementarias de investigación. La intención se vio también rodeada por aspectos económicos y sociales, los que fueron justificados para la creación de políticas educativas así como para impulsar la ampliación de la oferta educativa de la educación superior. El objetivo era obtener una mayor captación de población estudiantil, lo que a su vez permitió que en México los sectores de población que tradicionalmente no participaban en el ingreso a este tipo de instituciones educativas tuvieran la oportunidad de acceder a estos servicios y de tal forma se determinó un mecanismo efectivo de movilidad social. Fuentes (citado por Casillas y De Garay, 1992:23) explica esta relación entre el Estado y la sociedad como un intercambio político en el que los sectores demandantes de educación vieron satisfecha su solicitud y el Estado obtuvo consenso y legitimidad. Debido a que, de tal forma, distintos sectores sociales tienen la posibilidad de elevar sustancialmente su posición en la jerarquía social al acceder a la educación y encontrar empleos mejor remunerados, en correspondencia el Estado obtiene consenso y legitimidad entre los sectores beneficiados y ante la sociedad en su conjunto. En lo que respecta a la impartición de clases con calidad surge la flexibilidad curricular y 
las competencias profesionales dentro de las cuales los profesores de las IES han iniciado diversas tareas a desarrollar no solo en los niveles más altos de la educación sino que, asimismo, surgen la forma de adaptación y el comportamiento del docente con respecto a ciertas funciones sustantivas dentro de las instituciones de educación. Algunos autores afirman que a partir de la segunda mitad de la década de los noventa a la fecha, una de las políticas centrales para las instituciones públicas de educación superior ha sido la mejora de su calidad, tomando como base la formación académica del profesorado y su integración (Mota y Cisneros, 2009).

De acuerdo con las reglas de operación del Programa para el Desarrollo Profesional Docente (PRODEP), los así denominados Cuerpos Académicos (CA) se definen como “Un conjunto de profesores-investigadores que comparten una o más líneas de estudio, cuyos objetivos y metas están destinados a la generación y/o aplicación de nuevos conocimientos. Además, por el alto grado de especialización que alcanzan en conjunto al ejercer la docencia, logran una educación de buena calidad. Los cuerpos académicos sustentan las funciones académicas institucionales y contribuyen a integrar el sistema de educación superior del país" (Lara, 2010:142).

Otra conceptualización es la de Bajo y Martínez (2006) quienes los definen como “Grupos de profesores/as de tiempo completo que comparten una o varias líneas de generación de conocimiento, investigación aplicada o desarrollo tecnológico e innovación en temas disciplinares o multidisciplinares y un conjunto de objetivos y metas académicas. Adicionalmente atienden los programas educativos (PE) afines a su especialidad en varios tipos" (2006:2).

Otros elementos relacionados con los CA son las diferentes líneas de investigación. Las primeras (denominadas LGAC) se refieren a la generación o aplicación innovadora del conocimiento, que es una serie coherente de proyectos, actividades o estudios que profundizan en el conocimiento como producto de la investigación básica y aplicada, con un conjunto de objetivos y metas de carácter académico en temas disciplinares o multidisciplinares. Las segundas (denominadas LIIADT) contemplan las líneas innovadoras de investigación aplicada y desarrollo tecnológico, entendidas como una serie coherente de proyectos, actividades o estudios en temas disciplinares o multidisciplinares enfocados principalmente a la creación, desarrollo y mejora de tecnología con el fin de atender las necesidades del sector productivo y de servicios. Por último se encuentran las líneas de investigación en lengua, cultura y desarrollo (o LILCD), que comprenden una serie coherente de proyectos, actividades o estudios que profundizan en el conocimiento de la lengua, la cultura y el desarrollo de las culturas originarias, con un conjunto de objetivos y metas de carácter académico, en temas disciplinares o multidisciplinares (Secretaría de Gobernación-SEGOB, 2016:582).

Aquino, Medina y Escobedo (2013) apuntan que para determinar el nivel de desarrollo de un cuerpo académico el Programa para el Desarrollo Profesional Docente (PRODEP) lleva a cabo una evaluación de los productos académicos y de investigación generados durante un periodo de tres años. De acuerdo con dicha evaluación debe servir para interpretar, cambiar y mejorar las instituciones y programas educativos. La misma debe realizarse en forma permanente y participativa, además de ser un proceso abierto, flexible y establecido en el marco de la misión y de los objetivos de la institución. 
A partir de los resultados se otorga un estatus de acuerdo con la siguiente clasificación:

- Cuerpo Académico en Formación (CAEF). Los integrantes tienen definidas las LGAC, LIIADT o LILCD que cultivan. El CA tiene proyectos de investigación conjuntos para desarrollar las LGAC/LIIADT/LILCD. El CA tiene identificados a sus integrantes y algunos CA afines de otras instituciones del país o del extranjero con quienes desean establecer contactos.

- Cuerpo Académico en Consolidación (CAEC). Los productos de este CA deben ser productos académicos reconocidos por su calidad y que se deriven del desarrollo de las LGAC/LIIADT/LILCD que cultivan. Sus integrantes participan conjuntamente en LGAC/ LIIADT/LILCD bien definidas. Al menos la tercera parte de quienes lo integran contará con amplia experiencia en docencia y en formación de recursos humanos. Sus integrantes deben tener Reconocimiento al Perfil Deseable, el que es otorgado por la Subsecretaría de Educación Superior de la Secretaría de Educación Pública del Gobierno Federal a través del Programa para el Desarrollo Profesional Docente, a aquellos profesores de tiempo completo que cumplen satisfactoriamente las funciones universitarias y dan evidencia de ello por lo menos en los tres últimos años (SEGOB, 2016). El CAEC deberá contar con evidencias objetivas respecto a su vida colegiada y a las acciones académicas que realizan en colaboración entre sus integrantes, así como colaborar con otros CA.

- Cuerpo Académico Consolidado (CAC). Este debe contar con productos académicos reconocidos por su buena calidad y que se derivan de LGAC/LIIADT/LILCD consolidadas. Los integrantes del CA cuentan con amplia experiencia en docencia y en formación de recursos humanos. La mayoría de los integrantes cuenta con el Reconocimiento al Perfil Deseable. Asimismo tienen un alto compromiso con la institución, colaboran entre sí y su producción es evidencia de ello. “...mínimo deben ser tres integrantes y el número máximo está determinado por la comunicación e interacción eficaz y continua de sus miembros". (López, 2010:14).

Para alcanzar los estatus antes mencionados el Plan de Desarrollo Institucional de la Universidad Juárez Autónoma de Tabasco 2016-2020 contempla líneas estratégicas dirigidas a elevar la producción académica de los profesores. Para lograr esto deberá contarse con la capacidad de innovación continua de la institución, tanto en el ámbito académico como en la gestión, aunque el mayor peso radica en el personal docente calificado, el que debe estar en constante preparación y agrupado en cuerpos académicos. Debe promoverse la consolidación de dichos cuerpos académicos cuyas investigaciones coadyuven al progreso del propio sistema educativo y de la entidad ya que la investigación realizada permite la generación de conocimientos la que, a su vez, retroalimenta los procesos de docencia y vinculación. Por ello, mediante los cuerpos académicos y grupos de investigación la UJAT como Institución de Educación Superior (IES) explora e impulsa proyectos en los diferentes campos del conocimiento. "La importancia de los cuerpos académicos radica en que al desarrollarse un trabajo colegiado entre profesores de tiempo completo y de asignatura, para orientar y marcar el rumbo académico de la institución se impulsa la vinculación de los procesos para la generación y/o aplicación del conocimiento con sectores del exterior" (Alvarado, Manjarrez y Romero, 2010:7).

El presente estudio se enfocó en los cuerpos académicos cuyos miembros son Profesores de Tiempo Completo (PTC) de la División Académica de Ingeniería y Arquitectura (DAIA) de la Universidad Juárez Autónoma de Tabasco (UJAT), con el fin de conocer el nivel de desarrollo, evolución, factores de integración, sostenimiento y consolidación de los mismos. 
A partir del año 2004 la Universidad Juárez Autónoma de Tabasco inicia un proceso de evaluación de sus CA para generar información necesaria para el planeamiento institucional. Según el Informe de Actividades (UJAT, 2004), el total de cuerpos académicos para ese año era de 76 integrados por 772 profesores investigadores y 240 líneas de generación del conocimiento (LGAC). La mayoría de los cuerpos académicos se encontraban en formación y solo dos en proceso de consolidación. Derivado de los resultados de este diagnóstico se inician acciones encaminadas a elevar la productividad y habilitación de la planta docente. Para lograr lo anterior se establecieron compromisos en el marco de los Programas de Fortalecimiento Institucional (PIFI) impulsados por el Programa Nacional de Educación 2001-2006 que solicitó a todas las IES formular un PIFI que conjuntara y armonizara todas o gran parte de las acciones institucionales con la finalidad de impulsar la mejora continua y el aseguramiento de la calidad de los servicios que estas ofrecen (Sevilla, Galaz y Arcos, 2008). Entre los servicios a impulsar se encontraban la habilitación de la planta académica a grado preferente entendido como la obtención del grado de doctor, además de la adecuación de la infraestructura y la consolidación de los Cuerpos Académicos.

Tal como se muestra en la Figura 1, como resultado de las acciones llevadas a cabo para elevar la productividad de los profesores, en el año 2006 se obtuvo un incremento de dos CAEC a 10 CAEC. Estas cifras mostraron un incremento en lo sucesivo.

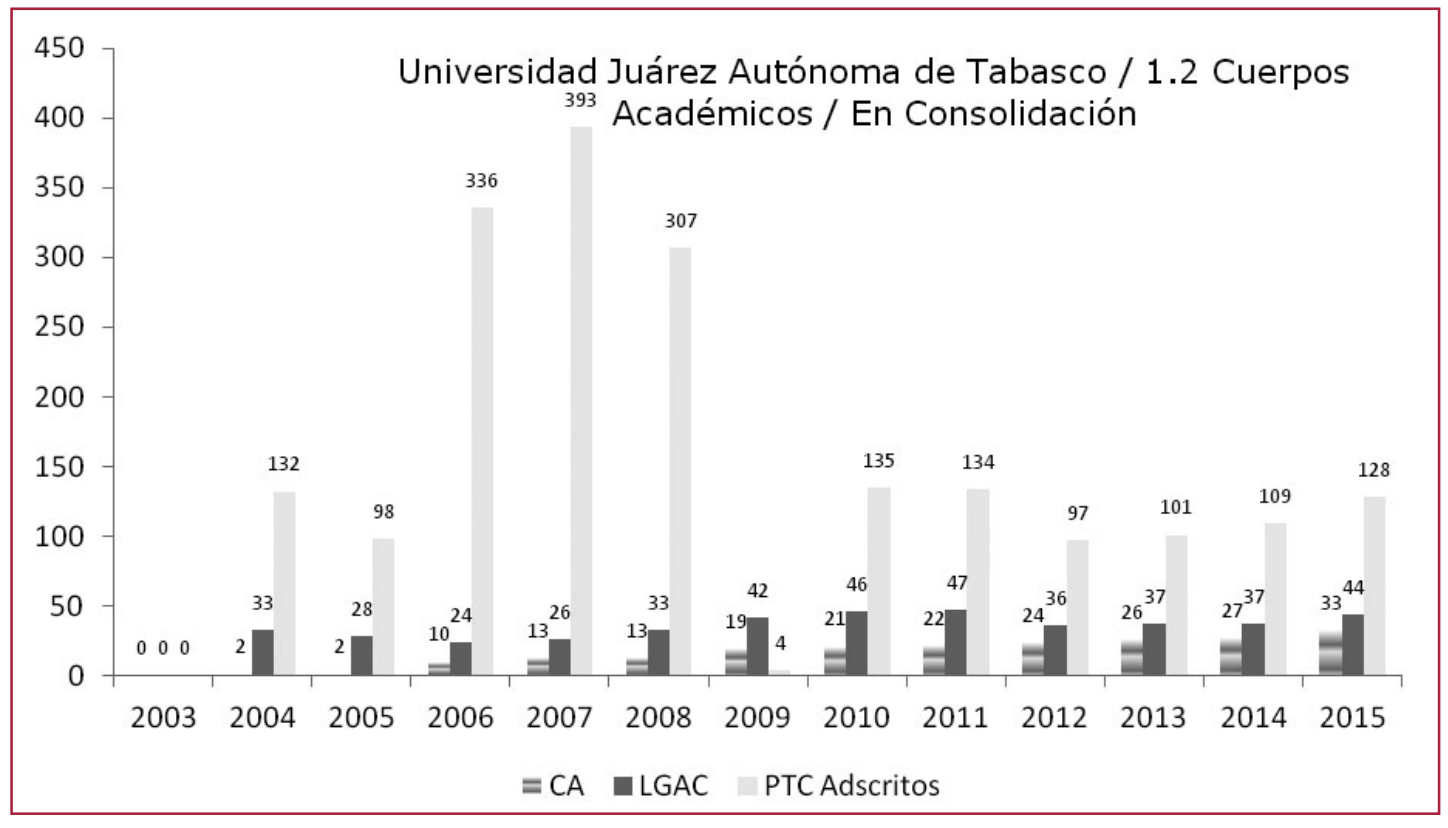

Figura 1. CAEC Fuente: Dirección de Planeación y Evaluación Institucional (2014).

Tal como puede en la Figura 2, en 2015 la UJAT cuenta con 63 cuerpos académicos. De estos, 11 están consolidados (CAC), 33 en consolidación (CAEC) y 19 en formación (CAEF) según dictamen emitido por el PRODEP. De esta manera el 70\% tiene reconocimiento de calidad lo que configura un avance sustancial comparado con el 38\% existente a principios de 2012. Con respecto a CAEF, estos han ido disminuyendo de un 58\% que estaban en formación en 2010 a un 30\% en 2015, mientras que el porcentaje de CAEC ha ido en aumento de un $36 \%$ en 2010 a 52\% en 2015. 


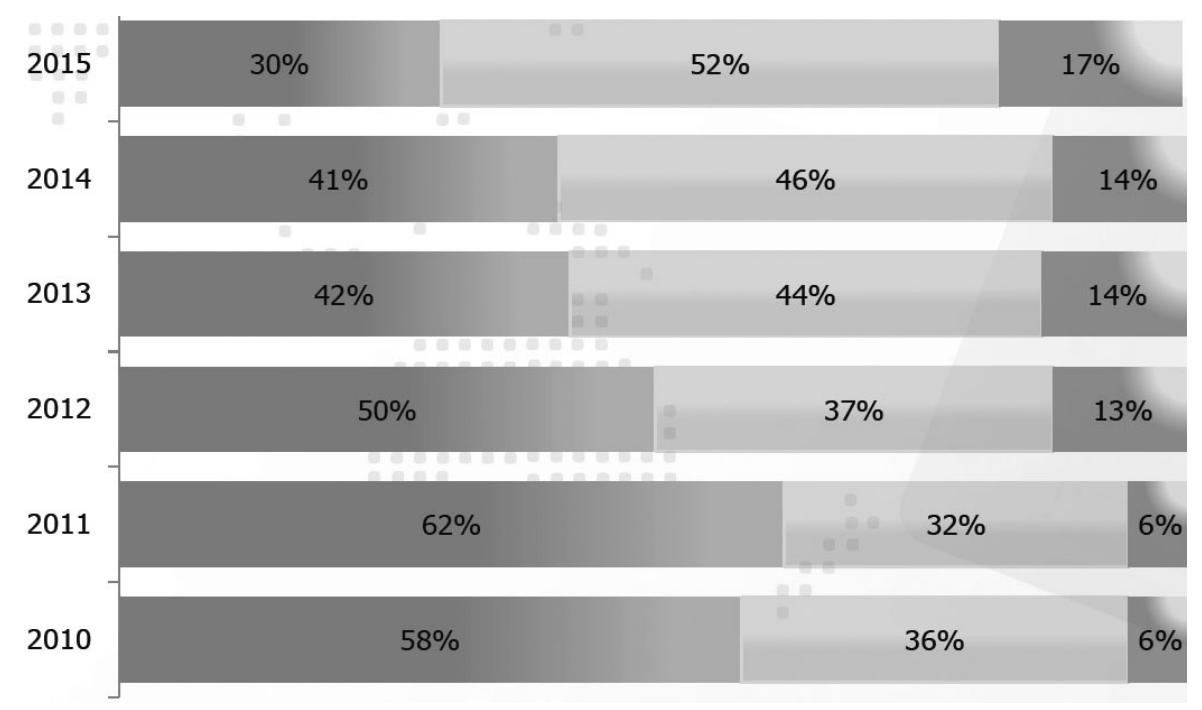

Figura 2. Evolución de los CA en la UJAT. Fuente: Plan de Desarrollo Institucional 2016-2020

Para incrementar el desarrollo de los CA de la UJAT fue necesario fomentar entre estos y los profesores la productividad académica colegiada, la cual va encauzada a las Líneas de Generación del Conocimiento (LGAC) cultivadas en la institución y para ello fue necesario aumentar la participación de los profesores en eventos nacionales e internacionales de calidad con publicaciones académicas y de investigación que estimularan la actualización en las áreas disciplinares, que favorecieran la productividad de los académicos y así contribuir a la difusión de su conocimiento así como al desarrollo intelectual del profesorado de cada una de las divisiones académicas. Para ello fue necesario partir de la situación en que cada facultad de la UJAT se encontraba en ese momento. Tal es el caso de la Facultad de Ingeniería y Arquitectura, que contó en 2005 con 13 CAF conformados por 85 profesores agrupados por áreas de conocimientos, quienes cultivaban 33 LGAC. Para ese entonces se contaba con un total de 158 profesores de los cuales solo un poco más del 53\% pertenecía a estas agrupaciones. Cabe mencionar que para esa fecha los CA estaban conformados por grupos disciplinares de acuerdo al área de estudios de los profesores y aún no se contaba con reglas de operación de los CA. Ante esto la Coordinación de Investigación de esta División Académica realiza un diagnóstico para elaborar el PIFI 3.0 y así plantear metas y gestionar recursos para el desarrollo de los CA y de los Programas Educativos adscritos.

La DAIA convocó a los integrantes de los grupos disciplinares para informar sobre los procesos de autoevaluación de cuerpos académicos y de participación en la elaboración PIFI cuya finalidad era la de concientizar a la planta académica de la división sobre la dimensión de los compromisos que implicaba pertenecer a los CA. Así es como en ese año en esta división académica se procedió a dar inicio a la elaboración de la autoevaluación indicada en el PIFI, solicitando a todos los integrantes de los antes llamados grupos disciplinares -ahora conocidos como Cuerpos Académicos- a llenar un formato para conocer el grado de dedicación a las actividades de docencia, gestión, investigación y tutoría. Los resultados en ese año mostraron que todos los profesores dedicaban la mayor parte del tiempo a impartir clases a grupos y no realizaban actividades de investigación ni tutoría y solo contaban con un mínimo de actividades relacionadas con gestión. Ante la inquietud de reforzar los CA se convocó a profesores que no formaban parte de ellos para que, acorde a su formación y especialización, colaboraran y se conformaran nuevos CA. Tal como lo 
indican Martínez, Rico y Preciado (2006, p.3), “La incorporación de un profesor a un CA debe ser libre y voluntaria, un profesor debe estar adscrito a un solo cuerpo aunque, desde luego, puede colaborar con otros".

En el apartado de resultados de esta investigación se describe la conformación y evolución de cada uno de ellos entre 2005 y 2015.

\section{Metodología}

La presente investigación está basada en un estudio descriptivo transversal con un enfoque de tipo mixto. La muestra fue no probabilística ya que la población analizada fue el total de cuerpos académicos de la División Académica de Ingeniería y Arquitectura, de los que se carece de registros históricos, desde la conformación hasta el estatus actual. En la primera etapa se revisaron los estudios propios de la institución relacionados con los cuerpos académicos. Posteriormente se analizaron datos estadísticos proporcionados por la Dirección General de Planeación y Evaluación Institucional (DGPEI) con el fin de conocer el número de CA, LGAC, integrantes y estatus. Con el fin de obtener información acerca de las experiencias propias de cada CA se empleó la entrevista estructurada como técnica de recolección de datos cualitativos dirigida a los líderes y ex líderes de cada CA. Dicha entrevista abordó cuatro categorías: a) antecedentes, b) conformación, c) evolución y d) debilidades y fortalezas de los cuerpos.

\section{Resultados}

Con la finalidad de analizar aspectos relacionados con antecedentes, conformación, evolución, debilidades y fortalezas de los CA en la DAIA se describen aspectos importantes para su desarrollo y el sostenimiento de cada uno de ellos. A continuación se describen:

\section{Cuerpo académico: innovación educativa - Ujat-Ca-180 Antecedentes}

Anteriormente estaba formado por un grupo de once profesores de tiempo completo pertenecientes a las siguientes áreas: dos de Ingeniería Química, tres de la Licenciatura en Idiomas, cuatro ingenieros civiles, uno de Licenciatura en Administración y uno de Licenciatura en Informática los cuales impartían materias de tronco común tales como Taller de Lectura y Redacción, Técnicas Administrativas, Lenguaje de Programación e Inglés. Cabe mencionar que ninguno de ellos contaba con posgrado y tampoco estaban involucrados en el desarrollo de proyectos de investigación. Asimismo desconocían las características de un CA o cómo debía conformarse para lograr los productos que se solicitaban. Ante ese desconocimiento realizaron una serie de reuniones de trabajo para definir el nombre de CA y designar al profesor responsable del grupo. El interés de los integrantes se centró en el análisis de problemas institucionales tales como formación docente de los profesores de ingeniería, didáctica de las ciencias, índices de reprobación, deserción y eficiencia terminal.

Fecha de creación: 2005

\section{Conformación}

Denominado CA Enseñanza en la Ingeniería ya que pretendía enfocarse en la realización de estudios relacionados con el análisis de los fenómenos educativos de la División Académica. De los once profesores que lo conformaban solo seis se comprometieron a realizar estudios de posgrado por lo que solo ellos continuaron. De los seis profesores que quedaban, uno de ellos trabajaba de manera aislada y uno pensaba realizar estudios de posgrado. Acorde a las recomendaciones de la Secretaria Académica este último fue dado de baja, quedando 
el grupo conformado por solo cuatro integrantes que trabajaron de manera colegiada durante los seis años siguientes. Posteriormente definieron las líneas de investigación de las cuales quedaron las siguientes: Diseño de Modelos Educativos para la Enseñanza de la Ingeniería y Gestión e Innovación en la Enseñanza de la Ingeniería. Los integrantes se involucraron en proyectos de investigación como colaboradores y concluyeron sus estudios de maestría obteniendo el grado en el área de ciencias de la educación, administración y sistemas de la información. Asimismo participaron en proyectos de investigación con financiamiento externo. Actualmente el CA lleva el nombre de Innovación Educativa y se encuentra en estatus de CAEC. En la Tabla 1 pueden visualizarse algunas de las limitantes encontradas en el periodo de conformación.

(Ver fortalezas y debilidades de este CA en Tabla 2).

\section{Cuerpo académico: materiales avanzados para ingeniería - Ujatab- Ca-187}

\section{Antecedentes}

Este CA inicia con cinco profesores, tres doctores de nueva contratación y dos PTC que ya se encontraban laborando en la División Académica que habían obtenido sus grados en Ingeniería de los Materiales, Química, Química de polímeros y Materiales.

Fecha de creación: 2006

\section{Conformación}

Uno de los PTC termina el doctorado en el año 2006; otro inicia sus estudios de doctorado en ese mismo año, lo que concluye en 2010. Los integrantes cultivan tres LGAC que son: Síntesis y Caracterización de Nuevos Materiales, Obtención de Materiales y Procesos para Control Ambiental e Investigación en Ciencia Aplicada. Actualmente este CA cuenta con cuatro integrantes. Debido a que no existían trabajos colegiados con uno de ellos, fue dado de baja. La Tabla 1 muestra algunas de las limitantes encontradas en el periodo de conformación.

(Ver fortalezas y debilidades de este CA en Tabla 2).

\section{Cuerpo académico: tecnologías electromecánicas teóricas y aplicadas - ujat-ca-205}

\section{Antecedentes}

Parte de este CA se encontraba trabajando dentro del grupo del área disciplinar de Ingeniería Mecánica con dos profesores que ya contaban con el grado preferente (doctor), dos con estudios de maestría en Ingeniería Mecánica y uno con estudios de licenciatura en Ingeniería Eléctrica. Este CA fue de los primeros en contar con el 90\% de sus integrantes con posgrado aunque carecían de actividades relacionadas con la investigación.

Fecha de creación: 2005

\section{Conformación}

Este CA no contaba con proyectos de investigación a pesar de estar ya integrado por profesores con estudios de doctorado en Ingeniería e Ingeniería Mecánica Eléctrica. Al principio cultivaban tres LGAC, de las cuales una fue eliminada debido a su improductividad. En el año 2006 el profesor que contaba solo con licenciatura termina sus estudios de maestría en Ingeniería Eléctrica. Actualmente este CA se encuentra trabajando con cuatro integrantes quienes cultivan dos LGAC: Integridad Mecánica y Diseño para Aplicaciones Industriales y Biomédicas y Tecnologías para uso Eficiente y Ahorro de Energía. En la Tabla 
1 pueden visualizarse algunas de las limitantes encontradas en el periodo de conformación. (Ver fortalezas y debilidades de este CA en Tabla 2).

\section{Cuerpo académico: procesos de ingeniería -ca ujat-ca-186 Antecedentes}

Este CA se integró con dos profesores de nueva contratación que contaban con grado preferente y un profesor investigador que se encontraba cursando sus estudios de posgrado.

Fecha de creación: 2005

\section{Conformación}

El CA cultiva solo una LGAC Ilamada Simulación, Modelado y Control de Sistemas de Ingeniería. En el año 2010 se integra otro miembro en calidad de colaborador integrante, quien finaliza sus estudios de doctorado en el año 2009.

En la Tabla 1 pueden visualizarse algunas de las limitantes encontradas en el periodo de conformación.

(Ver fortalezas y debilidades de este CA en Tabla 2).

\section{Cuerpo académico: ciencias de ingeniería y materiales - ujat-ca-185 Antecedentes}

Provenientes del grupo disciplinar de Ingeniería Química que se inicia con tres profesores que colaboraban como integrantes.

Fecha de Creación: 2005

\section{Conformación}

Dos de los tres profesores colaboradores obtienen su grado de doctor en el año 2007 especializándose en el área de Corrosión, Química Aplicada a la Ingeniería, y otro profesor con maestría en Ingeniería Química. Posteriormente se integra en el año 2013 un profesor de nueva contratación que contaba con el grado preferente en Ciencias de los Materiales. Este CA se encuentra cultivando la LGAC Desarrollo de Materiales para Control Ambiental y Evaluación de Materiales. En el año 2016 el profesor con maestría es dado de baja por jubilación.

En la Tabla 1 pueden observarse algunas de las limitantes encontradas en el periodo de conformación.

(Ver fortalezas y debilidades de este CA en Tabla 2).

\section{Cuerpo académico: optimización de sistemas electromecánicos - ca ujat-ca-55}

\section{Antecedentes}

Grupo disciplinar formado por seis profesores del área de Eléctrica y Electrónica y dos profesores del área de Ingeniería Mecánica Eléctrica. Se presentaron desavenencias con respecto a la toma del liderazgo del grupo, hecho que deterioró la relación entre sus integrantes. Ante esto se desintegró el mismo quedando solo tres profesores de la primera área.

Fecha de creación: 2005 


\section{Conformación}

CA conformado por tres profesores que pertenecían como colaboradores a uno de los grupos disciplinares del área de eléctrica y mecánica con estudios de maestría en Ingeniería en Control y Computación, Sistemas Eléctricos de Potencia y Electrónica. Las LGAC cultivadas fueron Mecatrónica Aplicada en Sistemas no Convencionales de Generación de Energía, Calidad de la Energía y Aplicaciones a los Sistemas Eléctricos-Electrónicos de Potencia. Durante su periodo de conformación se presentaron dificultades en la habilitación académica ya que solo uno de los miembros concluyó sus estudios doctorales aunque hasta la fecha no ha logrado titularse. Por tal razón, de momento ninguno de ellos cuenta con el grado preferente.

Ver en Tabla 1 las limitantes encontradas en el periodo de conformación de este CA. (Ver fortalezas y debilidades de este CA en Tabla 2).

\section{Cuerpo académico: hidráulica e hidrología -ca ujat-ca-56 Antecedentes}

Sus integrantes provienen del grupo disciplinar de ingeniería civil. La mayoría antes del año 2000 ya contaba con maestría. A pesar de ello carecían de productividad en investigación.

Fecha de creación: 2005

\section{Conformación}

Al llevarse a cabo la restructuración de los CA se forma este integrado por tres profesores, los tres con grado de maestría en Hidráulica obtenidos en 1988, 1990 y 1998 respectivamente. Realización de trabajos relacionados con sus dos LGAC en las áreas de Mecánica de Ríos, Hidrología Superficial y Subterránea. Durante su periodo de conformación presentaron ciertas dificultades. Actualmente cuenta con debilidades.

Ver Tabla 1.

(Ver fortalezas y debilidades de este CA en Tabla 2).

\section{Cuerpo académico: arquitectura y tecnología ambiental-ca ujat-ca-222 Antecedentes}

El 50\% de los integrantes de este CA se encontraba trabajando dentro del grupo del área disciplinar de arquitectura existente conformado por varios arquitectos que comenzaron sus estudios de maestría a partir de 2006. Dicho grupo se reestructuró en 2009 cuando algunos de sus integrantes todavía no habían obtenido el grado de maestría, por lo que muchos profesores de esta área desertaron de pertenecer al CA.

Fecha de creación: 2009

\section{Conformación}

Este CA se encontraba formado por dos profesores que pertenecían al grupo disciplinar del área de arquitectura y dos profesores investigadores que obtuvieron el grado de maestría en Arquitectura y Eco tecnología. Actualmente este CA cuenta con dos doctores en Arquitectura y Urbanismo y dos profesores que se encuentran realizando estudios de doctorado. Los mismos cultivan una LGAC Ilamada Arquitectura y Medioambiente.

En la Tabla 1 pueden visualizarse algunas de las limitantes encontradas en el periodo de conformación.

(Ver fortalezas y debilidades de este CA en Tabla 2). 


\section{Cuerpo académico sistemas eléctricos y electrónicos -ca ujat-ca-246 Antecedentes}

CA de nueva creación. Dos de sus colaboradores ingresan en 2011 y 2012 como PTC en la división.

Fecha de creación: 2015

\section{Conformación}

Conformado por dos profesores que pertenecían a un Grupo de Investigación y uno a un CA. En el mismo se encuentran colaborando tres doctores en Ingeniería Eléctrica e Ingeniería Electrónica. Actualmente su productividad está encaminada a una LGAC Ilamada Análisis de Control de Sistemas Eléctricos y Electrónicos.

En la Tabla 1 pueden visualizarse limitantes encontradas en el periodo de conformación. (Ver fortalezas y debilidades de este CA en Tabla 2).

Tabla 1. Limitantes durante la conformación de cada uno de los Cuerpos Académicos de la División Académica de Ingeniería y Arquitectura

\begin{tabular}{|c|c|}
\hline Cuerpo Académico & Dificultad \\
\hline \multirow[t]{2}{*}{ UJAT-CA-55 } & $\begin{array}{l}\text { Carencia de equilibrio entre las actividades de docencia, gestión, tutoría e } \\
\text { investigación. }\end{array}$ \\
\hline & Carencia de productividad en investigación \\
\hline UJAT-CA-56 & $\begin{array}{l}\text { Carencia de tiempo para poder equilibrar actividades de docencia, } \\
\text { investigación, tutorías y gestión académica }\end{array}$ \\
\hline \multirow{3}{*}{ UJAT-CA-180 } & $\begin{array}{l}\text { Falta de conocimiento para captura del currículum en el sistema en línea } \\
\text { de PROMEP }\end{array}$ \\
\hline & Falta de conocimiento para trabajar de manera colegiada \\
\hline & Saturación de horas frente a grupo \\
\hline UJAT-CA-185 & Carencia de actividad colegiada \\
\hline UJAT-CA-186 & Carencia de colaboración de índole internacional \\
\hline \multirow{3}{*}{ UJAT-CA-187 } & $\begin{array}{l}\text { Dificultades administrativas para adquirir los insumos de proyectos con } \\
\text { financiamiento. }\end{array}$ \\
\hline & $\begin{array}{l}\text { Falta de infraestructura para realizar una investigación de manera } \\
\text { consecutiva. }\end{array}$ \\
\hline & $\begin{array}{l}\text { Falta de equilibrio entre las actividades de docencia, gestión, tutoría e } \\
\text { investigación. }\end{array}$ \\
\hline \multirow[t]{2}{*}{ UJAT-CA-205 } & Existencia de varias líneas de generación de conocimiento \\
\hline & $\begin{array}{l}\text { Falta de equilibrio entre las actividades de docencia, gestión, tutoría e } \\
\text { investigación. }\end{array}$ \\
\hline UJAT-CA-222 & $\begin{array}{l}\text { Falta de participación de colaborador que se encontraba estudiando su } \\
\text { postgrado. }\end{array}$ \\
\hline UJAT-CA-246 & Cuerpo Académico de reciente creación \\
\hline
\end{tabular}

Fuente: Elaboración propia 
Tabla 2. Fortalezas y debilidades de cada uno de los Cuerpos Académicos de la División Académica de Ingeniería y Arquitectura

\begin{tabular}{|c|c|c|}
\hline $\begin{array}{l}\text { Cuerpo } \\
\text { Académico }\end{array}$ & Fortalezas & Debilidades \\
\hline \multirow[t]{3}{*}{ UJAT-CA-55 } & \multirow{2}{*}{$\begin{array}{l}100 \% \text { de sus integrantes } \\
\text { cuentan con el perfil deseable } \\
\text { Productividad académica } \\
\text { colegiada }\end{array}$} & $\begin{array}{l}\text { Ningún PTC pertenece al Sistema Nacional de } \\
\text { Investigadores (SNI) }\end{array}$ \\
\hline & & $\begin{array}{l}\text { Solo el } 25 \% \text { de los integrantes tiene el grado } \\
\text { preferente }\end{array}$ \\
\hline & Estatus en consolidación & $\begin{array}{l}\text { Carecen de participación colegiada en } \\
\text { Proyectos de Investigación con financiamiento }\end{array}$ \\
\hline \multirow[t]{5}{*}{ UJAT-CA-56 } & \multirow{5}{*}{$\begin{array}{l}\text { Todos sus integrantes cuentan } \\
\text { con el perfil deseable }\end{array}$} & Ningún PTC pertenece al SNI \\
\hline & & $\begin{array}{l}\text { Ninguno de los integrantes tiene el grado } \\
\text { preferente }\end{array}$ \\
\hline & & Falta de productividad académica colegiada \\
\hline & & $\begin{array}{l}\text { Carecen de participación colegiada y de } \\
\text { Proyectos de Investigación con financiamiento }\end{array}$ \\
\hline & & Estatus en formación \\
\hline \multirow[t]{6}{*}{ UJAT-CA-180 } & $\begin{array}{l}\text { Participación colegiada en } \\
\text { Proyectos de Investigación } \\
\text { internos }\end{array}$ & Formación de Recursos Humanos \\
\hline & $\begin{array}{l}\text { Participación colegiada en } \\
\text { Proyectos de Investigación con } \\
\text { financiamiento }\end{array}$ & Solo un PTC es SNI \\
\hline & $\begin{array}{l}\text { Todos sus integrantes cuentan } \\
\text { con el perfil deseable }\end{array}$ & \\
\hline & $\begin{array}{l}\text { La mayoría de sus integrantes } \\
\text { cuenta con título de doctorado }\end{array}$ & \\
\hline & $\begin{array}{l}\text { Productividad académica } \\
\text { colegiada }\end{array}$ & \\
\hline & Estatus en consolidación & \\
\hline \multirow[t]{4}{*}{ UJAT-CA-185 } & $\begin{array}{l}\text { Todos sus integrantes cuentan } \\
\text { con el perfil deseable }\end{array}$ & Solo un PTC es SNI \\
\hline & $\begin{array}{l}\text { Productividad académica } \\
\text { colegiada }\end{array}$ & \\
\hline & Estancias Académicas & \\
\hline & Estatus en consolidación & \\
\hline \multirow[t]{5}{*}{ UJAT-CA-186 } & $\begin{array}{l}100 \% \text { de sus integrantes tienen } \\
\text { el grado preferente }\end{array}$ & Solo un PTC es SNI \\
\hline & $\begin{array}{l}\text { Participación colegiada en } \\
\text { Proyectos de Investigación con } \\
\text { financiamiento }\end{array}$ & \\
\hline & $\begin{array}{l}\text { Todos sus integrantes cuentan } \\
\text { con el perfil deseable }\end{array}$ & \\
\hline & Estancias académicas & \\
\hline & Estatus consolidado & \\
\hline
\end{tabular}




\begin{tabular}{|c|c|c|}
\hline \multirow[t]{5}{*}{ UJAT-CA-187 } & $\begin{array}{l}\text { Todos los integrantes tienen el } \\
\text { grado preferente }\end{array}$ & \multirow[t]{5}{*}{ Solo un PTC es SNI } \\
\hline & $\begin{array}{l}\text { Participación colegiada en } \\
\text { Proyectos de Investigación con } \\
\text { financiamiento }\end{array}$ & \\
\hline & $\begin{array}{l}\text { Todos sus integrantes cuentan } \\
\text { con el perfil deseable }\end{array}$ & \\
\hline & $\begin{array}{l}\text { Productividad académica } \\
\text { colegiada }\end{array}$ & \\
\hline & Estatus en consolidación & \\
\hline \multirow[t]{4}{*}{ UJAT-CA-205 } & $\begin{array}{l}\text { Todos sus integrantes cuentan } \\
\text { con el perfil deseable }\end{array}$ & Ningún PTC es SNI \\
\hline & $\begin{array}{l}\text { Productividad académica } \\
\text { colegiada }\end{array}$ & $\begin{array}{l}\text { Solo el } 50 \% \text { de los integrantes tienen el grado } \\
\text { preferente }\end{array}$ \\
\hline & & $\begin{array}{l}\text { Carecen de participación colegiada en } \\
\text { Proyectos de Investigación con financiamiento }\end{array}$ \\
\hline & & Estatus en formación \\
\hline \multirow[t]{5}{*}{ UJAT-CA-222 } & $\begin{array}{l}50 \% \text { de sus integrantes tienen } \\
\text { el grado preferente }\end{array}$ & \multirow[t]{5}{*}{ Ninguno de sus integrantes es SNI } \\
\hline & $\begin{array}{l}\text { Participación colegiada en } \\
\text { Proyectos de Investigación con } \\
\text { financiamiento }\end{array}$ & \\
\hline & $\begin{array}{l}\text { Todos sus integrantes cuentan } \\
\text { con el perfil deseable }\end{array}$ & \\
\hline & $\begin{array}{l}\text { Se organizan para ejercer } \\
\text { el rol de líder del CA, cada } \\
\text { determinado periodo }\end{array}$ & \\
\hline & Estatus en consolidación & \\
\hline \multirow[t]{3}{*}{ UJAT-CA-246 } & $\begin{array}{l}\text { Todos sus integrantes tienen el } \\
\text { grado preferente }\end{array}$ & Ninguno de sus integrantes es SNI \\
\hline & $\begin{array}{l}\text { Participación colegiada en } \\
\text { Proyectos de Investigación con } \\
\text { financiamiento }\end{array}$ & \multirow[t]{2}{*}{ Estatus en formación } \\
\hline & $\begin{array}{l}\text { Todos sus integrantes cuentan } \\
\text { con el perfil deseable }\end{array}$ & \\
\hline
\end{tabular}

Fuente: Elaboración propia

Actualmente la División Académica de Ingeniería y Arquitectura cuenta con 206 profesores adscritos de los cuales 65 no pueden formar parte de los cuerpos académicos pues solo cuentan con estudios de licenciatura. Esto da como resultado un total de 141, de los cuales 32 forman parte de nueve cuerpos académicos que cultivan 14 LGAC.

En la Figura 3 puede observarse el decremento de los CAEF ya que de 12 CA que había en 2013 solo quedan tres siendo estos los CA de Optimización de Sistemas Electromecánicos, de Hidráulica e Hidrología y de Sistemas Eléctricos y Electrónicos. 


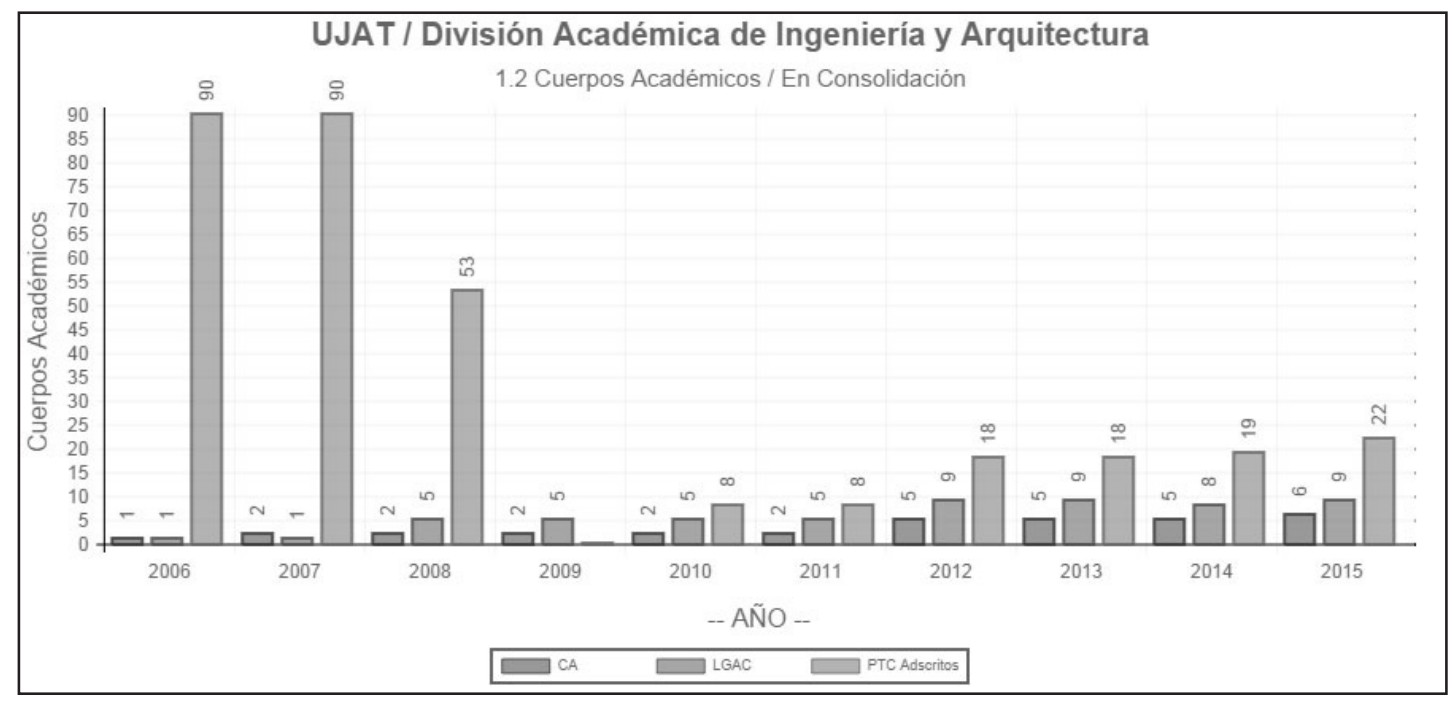

Figura 3. CAEF de la DAIA - Fuente: Informe de Actividades 2003 a 2014. Dir. General de Planeación y Evaluación Institucional

En la Figura 4 puede apreciarse que, con respecto a los CAEC, hubo un incremento de un CAEC en 2006 a seis en 2015. Cabe mencionar que el cambio de estatus se debe a los siguientes logros obtenidos: el porcentaje de los integrantes de estos CA cuenta con grado preferente, colaboración en proyectos con financiamiento, productividad académica colegiada cultivada acorde a sus LGAC e incremento de colaboradores con Reconocimiento al Perfil Deseable.

\section{UJAT / División Académica de Ingeniería y Arquitectura}

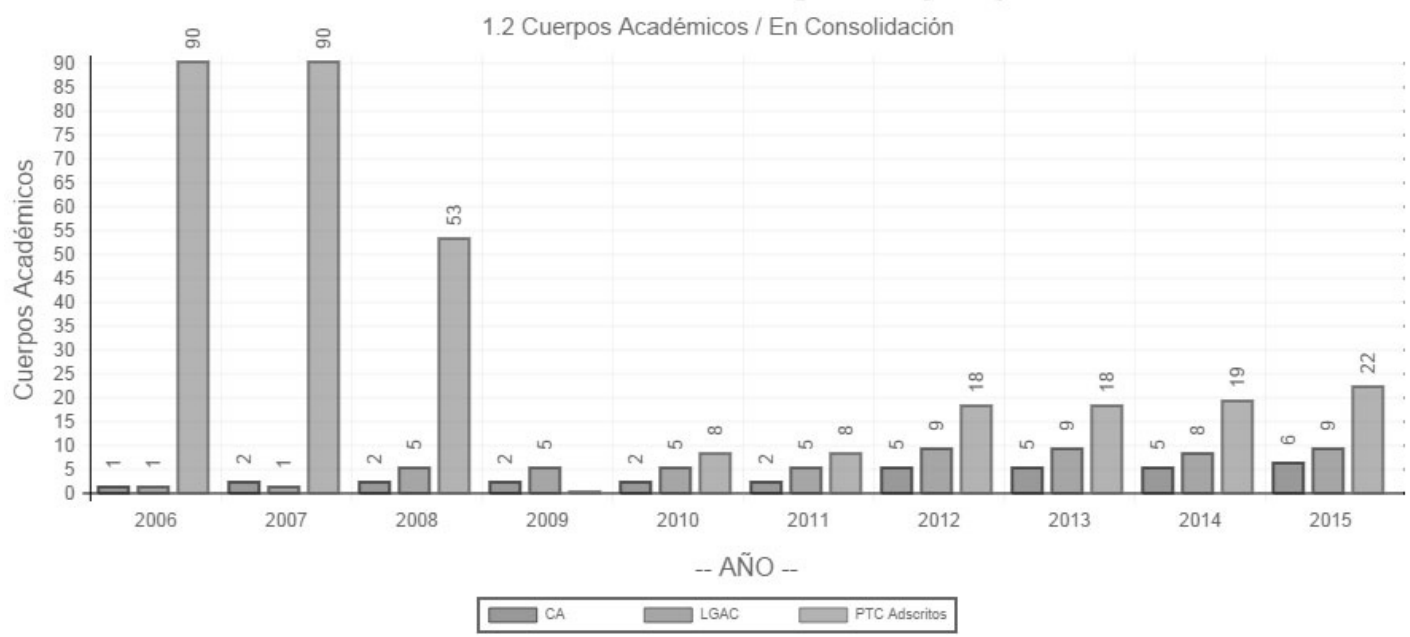

Figura 4: CAEC de la DAIA. Fuente: Informe de Actividades 2003 a 2014. Dir. General de Planeación y Evaluación Institucional 
Tal como se muestra en la Figura 5, en cuanto a los CAC de la división académica de Ingeniería y Arquitectura se sigue manteniendo igual que en el año 2008 habiendo solo uno en estatus consolidado.

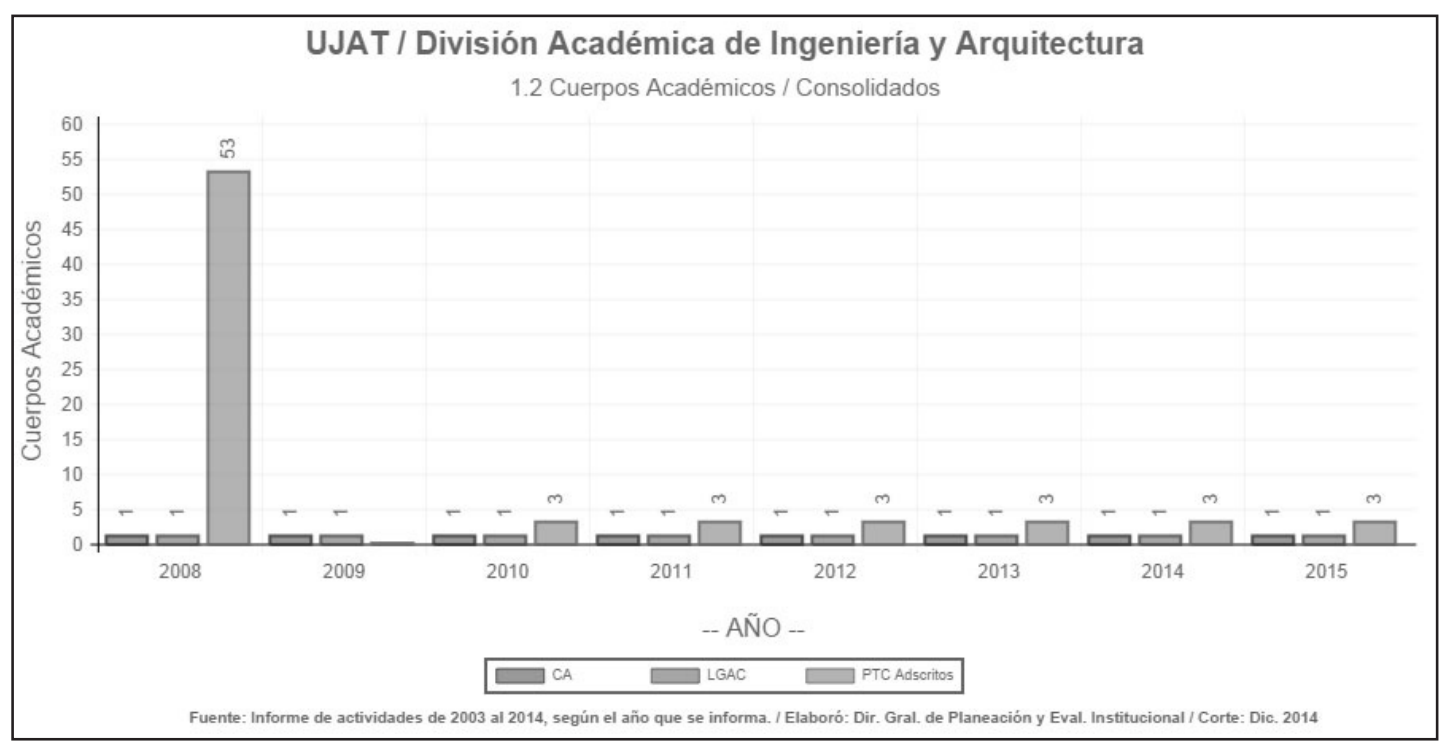

Figura 5: CAC de la DAIA. Fuente: Informe de Actividades 2003 a 2014. Dir. General de Planeación y Evaluación Institucional

\section{Conclusiones}

Dentro de los aspectos a destacar de los CA de la División Académica de Ingeniería y Arquitectura se identificó que menos del $50 \%$ de los profesores forman parte de cuerpos académicos y grupos de investigación. En cuanto a las actividades realizadas desde el año 2004 a la fecha se observó una evolución, ya que anteriormente no se desempeñaban actividades relacionadas con tutorías e investigación. Actualmente, más del 95\% de los profesores de tiempo completo que integran los cuerpos académicos realizan actividades de docencia, tutorías, investigación y gestión académica. El 90\% desarrolla proyectos de investigación con financiamiento, de los cuales se ha derivado una serie de productos colegiados, lo que ha permitido a más del $80 \%$ de los CA cambiar favorablemente de estatus al cumplir con las normativas de operación del Programa para el Desarrollo Profesional Docente.

Respecto a la evolución presentada por los CA es notorio el hecho de que hubo avances, principalmente hacia el status de En Consolidación con un incremento de uno que había en 2006 a seis en 2015.

En cuanto a las fortalezas que han influido para conformación de los CA pueden mencionarse las siguientes:

El 50\% de los integrantes cuenta con grado preferente

El $100 \%$ de los integrantes cuenta con el perfil deseable

Incremento en el número de productos académicos desarrollados entre los integrantes. Dichos productos consisten en: desarrollo de proyectos de investigación, publicación de artículos científicos, de libros y capítulos de libros, presentación de resultados de 
investigación en congresos, formación de recursos humanos a través de las direcciones de tesis y actualización de planes y programas de estudio.

Respecto a las debilidades, la mayor está representada por el escaso número de miembros del Sistema Nacional de Investigadores (SNI).

Por el contrario se observó que los CA de creación más reciente no presentan problemas en su conformación debido a que durante su integración las reglas de operación ya son más conocidas por otros CA, lo que ha permitido apoyarlos con sus experiencias y colaboración.

Dentro de las futuras líneas de investigación será necesario evaluar la contribución que han tenido los cuerpos académicos en la calidad de los programas educativos de la institución.

\section{Referencias bibliográficas}

Alvarado, V. M., Manjarrez, B. M. \& Romero, E. R. (2010). La calidad educativa y las competencias profesionales. En: La conformación de un cuerpo académico en la Escuela Nacional de Biblioteconomía y Archivonomía (México).

Aquino, Z. S. P., Medina, M. D. E. \& Escobedo, S. P. (2013). Cuerpos Académicos en educación superior. Retos para el desarrollo institucional. México, UJAT.

Bajo, A. y Martínez, R. (2006). Cuerpos académicos y desempeño institucional. El caso de la Universidad Autónoma de Sinaloa. México: Universidad Autónoma de Sinaloa.

Casillas, J. M. y De Garay, A. (1992). El contexto de la constitución del cuerpo académico en la educación superior 1960-1990. En: Académicos: un botón de muestra. México: Universidad Autónoma Metropolitana-Azcapotzalco.

Estrada, I. y Cisneros, E. (2009). Origen, reestructuración y desarrollo de los cuerpos académicos en una universidad pública del sureste de México. En: Memoria Electrónica, área 13 - Política y gestión Recuperado de http://www.comie.org.mx/congreso/ memoriaelectronica/v10/pdf/area_tematica_13/ponencias/0528-F.pdf

Lara, R. J. J. (2010). Once experiencias y perspectivas en la integración de redes temáticas de colaboración de cuerpos académicos. Redes de conocimiento en el noroeste de México. En:J. B. Castañeda Cortés (Coordinador). Los cuerpos académicos del PROMEP. Una valoración de su política y el trabajo en redes. Tomo I, Culiacán, Sinaloa: UAS/Ediciones del Lirio, 07-284

López, L. S. (2010). Cuerpos académicos: factores de integración y producción de conocimiento, Revista de la educación superior, 155, 7-25.

Martínez, P. C., Rico, R. M. y Preciado, S. E. (2006). Evolución de los cuerpos académicos en la Universidad de Guanajuato. Recuperado de http://www.redalyc.org/pdf/416/41600302.pdf

Mota, I. E. y Cisneros, C. E. (2009). Origen, reestructuración y desarrollo de los cuerpos académicos en una universidad pública del sureste de México, en Memoria Electrónica, área 13, Recuperado de http://www.comie.org.mx/congreso/memoriaelectronica/v10/pdf/ area_tematica_13/ponencias/0528-F.pdf 
Secretaría de Gobernación [SEGOB], (2016). Acuerdo número 21/216 por el que se emiten las Reglas de Operación del Programa para el Desarrollo Profesional Docente para el Ejercicio Fiscal 2017. Diario Oficial de la Federación. Séptima sección. Recuperado de http:// www.dof.gob.mx/nota_detalle.php?codigo=5467922\&fecha=28/12/2016

Sevilla, J. J., Galaz F. \& Arcos, J. L. (2008). La participación del académico en procesos de planeación y su visión institucional. Revista electrónica de investigación educativa, 10(2).

Universidad Juárez Autónoma de Tabasco [UJAT]. (2016). Plan de Desarrollo Institucional. (2016-2020). Recuperado de http://www.archivos.ujat.mx/2016/rectoria/PDI FINAL2020.pdf

Universidad Juárez Autónoma de Tabasco [UJAT], (2004) Informe de Actividades División Académica de Ingeniería y Arquitectura. http://infohistorica.ujat.mx/

Universidad Juárez Autónoma de Tabasco [UJAT], (2014). 2do. Informe de Actividades División Académica de Ingeniería y Arquitectura 2014. Dr. Candelario Bolaina Torres. Tabasco, México. 